\title{
Fractality in hadron interactions: a conservation law and quantization of fractal dimensions
}

\author{
Imrich Zborovský1,* \\ ${ }^{1}$ Nuclear Physics Institute of the CAS, 25068 Řež, Czech Republic
}

\begin{abstract}
A microscopic scenario of constituent interactions in high energy collisions of hadrons and nuclei is studied. The concept is based on the $z$-scaling of inclusive spectra observed in a wide range of collision energies, multiplicity densities, momenta, and angles of detected particles. We bring arguments that, due to the fractality and self-similarity of the hadron interactions assumed in the $z$-scaling scheme, there exists a conservation law of a new quantity named "fractal cumulativity." The conserved quantity is proportional to the corresponding fractal dimensions and is a simple function of the respective momentum fractions. Based on statistical ideas and entropy considerations we demonstrate that the fractal dimensions possess quantum character.
\end{abstract}

\section{Introduction}

Universal principles are usually reflected as regularities in measured observables. They can be expressed as scalings in different representations of data. One of the scaling behavior related to the principles of self-similarity and fractality of hadron interactions is manifested by the $z$-scaling (see [1] and references therein). The method of $z$-scaling was applied in proton-(anti)proton collisions for analysis of the inclusive spectra of different hadrons including heavy quarkonia and top quark [2-5]. The scaling hypothesis was tested in relativistic collisions of heavy nuclei using $z$-presentation of the transverse momentum distributions of charged hadrons and pions [6-12]. The observed regularity is interpreted as a manifestation of the self-similarity of hadron interactions at a constituent level and is considered as an expression of fractality of the colliding objects and processes of fragmentation into real particles [13].

The scaling variable $z$ is a fractal measure reflecting symmetries and quantum properties of the interacting fractal systems. We bring arguments that, due to the fractality and selfsimilarity of hadron interactions assumed in the $z$-scaling scheme, there exists a conservation law of a new quantity named "fractal cumulativity" [14]. In the initial state, the quantity characterizes a cumulative property of the internal fractal structures of the colliding hadrons or nuclei. The cumulativity in the final state reflects aggregation property of the fragmentation processes by the production of the inclusive particle and its recoiling counterpart. The conserved quantity is proportional to the corresponding fractal dimensions and is a simple function of the respective momentum fractions. Based on statistical ideas and entropy considerations we demonstrate that the fractal dimensions possess quantum character. This results

\footnotetext{
*e-mail: zborovsky@ujf.cas.cz
} 
in quantization of the fractal cumulativity. It follows that, assuming the fractal self-similarity of hadron interactions, the number of quanta of the fractal cumulativity is preserved.

\section{2 z-Scaling}

At sufficiently high energies, the collision of extended objects like hadrons and nuclei is considered as an ensemble of individual interactions of their constituents. The constituents are partons in the parton model or quarks and gluons in the theory of QCD. Structures of the colliding objects are characterized by parameters $\delta_{1}$ and $\delta_{2}$. The constituents of the incoming hadrons (or nuclei) with masses $M_{1}, M_{2}$ and momenta $P_{1}, P_{2}$ carry their fractions $x_{1}, x_{2}$. The inclusive particle carries the momentum fraction $y_{a}$ of the scattered constituent with a fragmentation characterized by a parameter $\varepsilon_{a}$. A fragmentation of the recoil constituent is described by $\varepsilon_{b}$ and the momentum fraction $y_{b}$. Multiple interactions are considered as similar. It represents the self-similarity of hadron interactions at a constituent level.

\subsection{Momentum fractions $x_{1}, x_{2}, y_{a}, y_{b}$ and scaling variable $z$}

The idea of the $z$-scaling is based on the assumption that gross features of an inclusive particle distribution of the reaction

$$
M_{1}+M_{2} \rightarrow m_{a}+X
$$

can be described at high energies in terms of the kinematic characteristics of a corresponding constituent subprocess. We consider the subprocess to be a binary collision

$$
\left(x_{1} M_{1}\right)+\left(x_{2} M_{2}\right) \rightarrow\left(m_{a} / y_{a}\right)+\left(x_{1} M_{1}+x_{2} M_{2}+m_{b} / y_{b}\right)
$$

of the constituents with masses $\left(x_{1} M_{1}\right)$ and $\left(x_{2} M_{2}\right)$ resulting in the scattered and recoil objects in the final state with masses $\left(m_{a} / y_{a}\right)$ and $\left(x_{1} M_{1}+x_{2} M_{2}+m_{b} / y_{b}\right)$, respectively. The registered particle with mass $m_{a}$ and the 4-momentum $p$ carries the fraction $y_{a}$ of the 4-momentum of the scattered constituent. Its hadron counterpart with mass $m_{b}$, moving in the opposite direction, carries the 4-momentum fraction $y_{b}$ of the produced recoil. The momentum conservation law in the constituent subprocess is connected with the recoil mass $M_{X}=\left(x_{1} M_{1}+x_{2} M_{2}+m_{b} / y_{b}\right)$ of the recoil system as follows

$$
\left(x_{1} P_{1}+x_{2} P_{2}-p / y_{a}\right)^{2}=M_{X}^{2} .
$$

This equation represents a kinematic constraint for the momentum fractions $x_{1}, x_{2}, y_{a}$, and $y_{b}$ which determine the subprocess (2).

Fractal structures of the colliding hadrons (or nuclei) and fragmentation of the objects produced in the scattered and recoil directions are described by the fractal dimensions $\delta_{1}, \delta_{2}$, and $\varepsilon_{a}, \varepsilon_{b}$, respectively. We connect the structural parameters with the corresponding momentum fractions by the function [1]

$$
\Omega\left(x_{1}, x_{2}, y_{a}, y_{b}\right)=\left(1-x_{1}\right)^{\delta_{1}}\left(1-x_{2}\right)^{\delta_{2}}\left(1-y_{a}\right)^{\varepsilon_{a}}\left(1-y_{b}\right)^{\varepsilon_{b}} .
$$

Physical interpretation of $\Omega$ is given by its proportionality to the relative number of all such constituent configurations in the reaction (1) which contain the configuration defined by the fractions $x_{1}, x_{2}, y_{a}$ and $y_{b}$. The function $\Omega$ plays the role of a relative volume occupied by these configurations in the space of the momentum fractions. The scaling variable

$$
z=z_{0} \Omega^{-1}
$$


is a fractal measure which diverges with the increasing resolution $\Omega^{-1} \rightarrow \infty$. The $\Omega$ is maximal value of the function (4) under the condition (3). The finite part $z_{0}=$ $\sqrt{s_{\perp}} /\left[\left(d N_{c h} /\left.d \eta\right|_{0}\right)^{c} m_{N}\right]$ of the self-similarity scaling variable is proportional to the transverse kinetic energy $\sqrt{s_{\perp}}$ of the underlying constituent subprocess (2) consumed on production of the inclusive particle with the 4-momentum $p$ and its recoiling counterpart with mass $m_{b}$. The multiplicity density of charged particles in the central region $\left(d N_{c h} /\left.d \eta\right|_{0}\right)$ is raised to the power of $c$, interpreted as a "specific heat" (see Eqs. (6) - (10)), and $m_{N}$ is the nucleon mass.

\subsection{Variable $z$ and entropy}

There exists a profound connection between the variable $z$ and entropy [1]. The scaling variable is proportional to the ratio

$$
z \sim \frac{\sqrt{s_{\perp}}}{W}
$$

of the transverse kinetic energy $\sqrt{s_{\perp}}$ and the maximal value of the function

$$
W\left(x_{1}, x_{2}, y_{a}, y_{b}\right)=\left(d N_{c h} /\left.d \eta\right|_{0}\right)^{c} \Omega\left(x_{1}, x_{2}, y_{a}, y_{b}\right)
$$

satisfying the condition (3). According to statistical physics, the entropy of a system is given by the number $W_{s}$ of its statistical states as follows

$$
S=\ln W_{s}
$$

The most likely configuration of the system is given by the maximal value of $S$. For inclusive reactions, the quantity $W_{s}$ is the number of all parton and hadron configurations in the initial and final state of the colliding system which can contribute to the production of the inclusive particle with a given momentum $p$. The configurations comprise all constituent configurations that are mutually connected by the independent subprocesses (2). The subprocesses are subject to the condition (3). The underlying subprocess, which defines the variable $z$, is singled out from the subprocesses by the principle of maximal entropy $S$. The absolute number of the corresponding configurations $W_{s}=W W_{0}$ is given up to a multiplicative constant $W_{0}$. Its value is restricted by the positiveness of entropy above some scale characterized by a maximal resolution $\left(\Omega^{-1}\right)_{\max }$. For the infinite resolution at fractal limit, $W_{0}$ is classically infinity.

In thermodynamics, the entropy of an ideal gas is given by the formula

$$
S=c_{V} \ln T+R \ln V+S_{0},
$$

where $c_{V}$ is a specific heat and $R$ is an universal constant. The temperature $T$ and the volume $V$ characterize a state of the system. Using the relations (4) and (7) - (9), one obtains

$$
S=c \ln \left[d N_{c h} /\left.d \eta\right|_{0}\right]+\ln \left[\left(1-x_{1}\right)^{\delta_{1}}\left(1-x_{2}\right)^{\delta_{2}}\left(1-y_{a}\right)^{\varepsilon_{a}}\left(1-y_{b}\right)^{\varepsilon_{b}}\right]+\ln W_{0} .
$$

We refer to the analogy between Eqs. (9) and (10). The analogy is supported by the plausible idea that the interactions of the extended objects like hadrons and nuclei can be treated at sufficiently high energies as a set of independent collisions of their constituents. Such a concept justifies the division of the system into the part comprising the subprocesses which can contribute to the production of the inclusive particle with the 4-momentum $p$ and the rest of the system containing all remaining microscopic configurations which lead to the produced multiplicity. 


\section{Conservation of fractal cumulativity}

The requirement of the minimal resolution $\Omega^{-1}$ of the fractal measure $z$ with respect to all constituent subprocesses (2) with the constraint (3) is equivalent to the principle of the maximal entropy (10) of the rest of the system. The conditions for maximization of the entropy $S\left(x_{1}, x_{2}, y_{a}, y_{b}\right)$ define the momentum fractions which correspond to the minimal necessary resolution needed to single out such a subprocess. The momentum fractions obtained in this way satisfy the following equality [14]

$$
\frac{\delta_{1} x_{1}}{\left(1-x_{1}\right)}+\frac{\delta_{2} x_{2}}{\left(1-x_{2}\right)}=\frac{\varepsilon_{a} y_{a}}{\left(1-y_{a}\right)}+\frac{\varepsilon_{b} y_{b}}{\left(1-y_{b}\right)}
$$

This equation represents a conservation law for the quantity

$$
C(D, \zeta)=D g(\zeta), \quad g(\zeta)=\frac{\zeta}{(1-\zeta)}
$$

The symbol $D\left(=\delta_{1}, \delta_{2}, \varepsilon_{a}, \varepsilon_{b}\right)$ means a fractal dimension and $\zeta\left(=x_{1}, x_{2}, y_{a}, y_{b}\right)$ is the corresponding momentum fraction. The conservation law (11) holds for any inclusive reaction (1) with arbitrary momenta $P_{1}, P_{2}$ and $p$ of the colliding and inclusive particles.

We call the conserved quantity $C(D, \zeta)$ as "cumulativity" or more precisely "fractal cumulativity" of a fractal-like object (or a fractal-like process) with a fractal dimension $D$. The quantity reflects ability of fractal systems to form structural aggregates with a certain degree of local compactness. The cumulativity of the internal structures of the interacting objects is proportional to $\delta_{1}$ and $\delta_{2}$, respectively. The cumulativity of the fragmentation processes in the scattered (recoil) direction is proportional to $\varepsilon_{a}\left(\varepsilon_{b}\right)$. The parameters characterize the fractal nature of the hadron compositeness and reflect the fractal properties of the fragmentation processes in the final state, respectively. The scale dependence of the cumulativity $C(D, \zeta)$ is given by the function $g(\zeta)$. It takes the same form for all considered interacting objects (different hadrons, nuclei, hadron constituents, jets, guarks, gluons etc.). The fractal cumulativity of the interacting hadron constituents is large when they carry large fractions of momenta of the structural objects they belong to. This property is conserved in hadron interactions and is transmitted to the scattered and recoil systems, which fragment into the inclusive particle and its hadron counterpart, respectively. The fractal structure characterized by the small cumulativities contains only constituents that contribute with small portions to its momentum.

\section{Quantization of fractal dimensions}

The fractality of hadron structure and fragmentation processes manifests itself most prominently near the kinematic limit of the reaction (1). The entropy of the constituent configurations in this region bears information on fractal characteristics of the hadron interactions at small distances. In the vicinity of the kinematic limit (i.e. near the fractal limit), the entropy can be expressed in a specific form which allows to draw some physical conclusions. We will demonstrate that there are terms in the formula for the entropy that admit physical interpretation of a quantization of the fractal dimensions $\delta_{1}, \delta_{2}, \varepsilon_{a}$ and $\varepsilon_{b}$. In order to show this explicitly, let us focus on the momentum dependent part, $S_{\Omega}$, of the expression (10), which has the form

$$
S_{\Omega}=\delta_{1} \ln \left(1-x_{1}\right)+\delta_{2} \ln \left(1-x_{2}\right)+\varepsilon_{a} \ln \left(1-y_{a}\right)+\varepsilon_{b} \ln \left(1-y_{b}\right)+\ln \Omega_{0} .
$$


For the infinite resolution $\Omega^{-1}$, all momentum fractions become unity $\left(x_{1}=x_{2}=y_{a}=y_{b}=1\right)$. This kinematic limit corresponds to the fractal limit $z=\infty$. The momentum fractions can be approximated near the fractal limit as follows

$$
\begin{array}{ll}
1-x_{1}=\frac{\left(1-e_{1}-e_{2}\right)}{\left(\bar{\lambda}_{1}+\bar{\lambda}_{0}\right)} \frac{\delta_{1}}{\delta+\varepsilon}, & 1-y_{a}=\frac{\left(1-e_{1}-e_{2}\right)}{(1-v)} \frac{\varepsilon_{a}}{\delta+\varepsilon}, \\
1-x_{2}=\frac{\left(1-e_{1}-e_{2}\right)}{\left(\bar{\lambda}_{2}+\bar{\lambda}_{0}\right)} \frac{\delta_{2}}{\delta+\varepsilon}, & 1-y_{b}=\frac{\left(1-e_{1}-e_{2}\right)}{\left(v+\bar{\lambda}_{0}\right)} \frac{\varepsilon_{b}}{\delta+\varepsilon},
\end{array}
$$

where $\delta \equiv \delta_{1}+\delta_{2}$ and $\varepsilon \equiv \varepsilon_{a}+\varepsilon_{b}$ [14]. The above expressions are linear functions of the combination $e_{1}+e_{2}$ that depends on the transverse momentum $p_{T}$ of the inclusive particle. The constants $\bar{\lambda}_{0}$ and $v$ are functions of the masses $M_{1}, M_{2}, m_{a}, m_{b}$, and the total energy $\sqrt{s}$. The angular dependent quantities $\bar{\lambda}_{1}$ and $\bar{\lambda}_{2}$ are calculated at the kinematic boundary of the reaction (1). Using Eqs. (14) and (15), the entropy $S_{\Omega}$ can be rewritten near the fractal limit as follows

$$
\begin{aligned}
S_{\Omega} & =(\delta+\varepsilon) \ln \left(1-e_{1}-e_{2}\right)+\ln \Omega_{0}-S_{\Gamma} \\
& -\delta_{1} \ln \left(\bar{\lambda}_{1}+\bar{\lambda}_{0}\right)-\delta_{2} \ln \left(\bar{\lambda}_{2}+\bar{\lambda}_{0}\right)-\varepsilon_{a} \ln (1-v)-\varepsilon_{b} \ln \left(v+\bar{\lambda}_{0}\right),
\end{aligned}
$$

where

$$
\begin{aligned}
S_{\Gamma} & =\delta_{1}\left[\left(1+\frac{\delta_{2}}{\delta_{1}}\right) \ln \left(1+\frac{\delta_{2}}{\delta_{1}}\right)-\frac{\delta_{2}}{\delta_{1}} \ln \frac{\delta_{2}}{\delta_{1}}\right]+\varepsilon_{a}\left[\left(1+\frac{\varepsilon_{b}}{\varepsilon_{a}}\right) \ln \left(1+\frac{\varepsilon_{b}}{\varepsilon_{a}}\right)-\frac{\varepsilon_{b}}{\varepsilon_{a}} \ln \frac{\varepsilon_{b}}{\varepsilon_{a}}\right] \\
& +\delta\left[\left(1+\frac{\varepsilon}{\delta}\right) \ln \left(1+\frac{\varepsilon}{\delta}\right)-\frac{\varepsilon}{\delta} \ln \frac{\varepsilon}{\delta}\right] .
\end{aligned}
$$

Let us consider the entropy contribution $S_{\Gamma}$ that depends on fractal dimensions only. It can be interpreted in terms of a statistical ensemble of the internal fractal configurations of the colliding hadrons (or nuclei) and the fractal configurations corresponding to the fragmentation processes in the final state. Let us suppose that the entropy of a single "average" fractal configuration of the system reads

$$
S_{\mathrm{I}}\left(\frac{\delta_{2}}{\delta_{1}}\right)+S_{\mathrm{I}}\left(\frac{\varepsilon_{b}}{\varepsilon_{a}}\right)+S_{\mathrm{I}}\left(\frac{\varepsilon}{\delta}\right),
$$

where $S_{\mathrm{I}}(r)=d[(1+r) \ln (1+r)-r \ln r]$ is proportional to a constant $d$. The statistical ensemble of the interacting fractal configurations is considered as a large collection of $n_{\delta_{1}}$ identical fractals with random configurations but with the same fractal dimension $\delta_{1}$ interacting with analogous large set of $n_{\delta_{2}}$ identical fractals with the fractal dimension $\delta_{2}$ which results in another large collection of $n_{\varepsilon_{a}}$ identical fractals with random configurations but with the same fractal dimension $\varepsilon_{a}$ and the corresponding large collection of $n_{\varepsilon_{b}}$ identical fractals with the fractal dimension $\varepsilon_{b}$. The entropy $S_{\Gamma}$ for the whole statistical ensemble is

$$
S_{\Gamma}=n_{\delta_{1}} S_{\mathrm{I}}\left(\frac{\delta_{2}}{\delta_{1}}\right)+n_{\varepsilon_{a}} S_{\mathrm{I}}\left(\frac{\varepsilon_{b}}{\varepsilon_{a}}\right)+\left(n_{\delta_{1}}+n_{\delta_{2}}\right) S_{\mathrm{I}}\left(\frac{\varepsilon}{\delta}\right) .
$$

The relation allows us to draw some physical consequences provided the fractal dimensions are expressed as integer multiples of the same constant $d$ :

$$
\delta_{1}=d \cdot n_{\delta_{1}}, \quad \delta_{2}=d \cdot n_{\delta_{2}}, \quad \varepsilon_{a}=d \cdot n_{\varepsilon_{a}}, \quad \varepsilon_{b}=d \cdot n_{\varepsilon_{b}} .
$$

Using the fractal dimensions in the above quantum form, we can write

$$
S_{\Gamma}=n_{\delta_{1}} S_{\mathrm{I}}\left(\frac{n_{\delta_{2}}}{n_{\delta_{1}}}\right)+n_{\varepsilon_{a}} S_{\mathrm{I}}\left(\frac{n_{\varepsilon_{b}}}{n_{\varepsilon_{a}}}\right)+n_{\delta} S_{\mathrm{I}}\left(\frac{n_{\varepsilon}}{n_{\delta}}\right),
$$


where $n_{\delta} \equiv n_{\delta_{1}}+n_{\delta_{2}}$ and $n_{\varepsilon} \equiv n_{\varepsilon_{a}}+n_{\varepsilon_{b}}$. According to statistical physics, this gives a possibility to interpret the entropy $S_{\Gamma}$ as the logarithm of number of ways in which the fractal dimensions of the interacting fractal structures can be composed from the identical dimensional quanta, each of the size $d$. The overall number of the dimensional quanta, $n=n_{\delta}+n_{\varepsilon}$, corresponding to a specific realization of the reaction (1) is distributed between $n_{\delta}=n_{\delta_{1}}+n_{\delta_{2}}$ quanta of fractal dimensions in the initial state and $n_{\varepsilon}=n_{\varepsilon_{a}}+n_{\varepsilon_{b}}$ dimensional quanta in the final state. The possible different arrangements of such a distribution amounts to

$$
\Gamma_{\delta, \varepsilon}=\frac{\left(n_{\delta}+n_{\varepsilon}\right) !}{n_{\delta} ! n_{\varepsilon} !}
$$

The symbol $\Gamma_{\delta, \varepsilon}$ represents the number of the different ways how $n_{\delta}+n_{\varepsilon}$ identical dimensional quanta can be shared among $n_{\delta}$ portions pertaining to the fractal dimensions of the colliding objects and $n_{\varepsilon}$ portions belonging to the fractal dimensions characterizing the fractal structures in the final state. The number of the different ways in which the numbers $n_{\delta}=n_{\delta_{1}}+n_{\delta_{2}}$ and $n_{\varepsilon}=n_{\varepsilon_{a}}+n_{\varepsilon_{b}}$ of dimensional quanta can be distributed between the fractal dimensions of the single interacting fractals is given as follows

$$
\Gamma_{\delta_{1}, \delta_{2}}=\frac{\left(n_{\delta_{1}}+n_{\delta_{2}}\right) !}{n_{\delta_{1}} ! n_{\delta_{2}} !}, \quad \Gamma_{\varepsilon_{a}, \varepsilon_{b}}=\frac{\left(n_{\varepsilon_{a}}+n_{\varepsilon_{b}}\right) !}{n_{\varepsilon_{a}} ! n_{\varepsilon_{b}} !}
$$

Expressed in terms of the dimensional constant $d$, the corresponding entropy is

$$
\tilde{S}_{\Gamma}=d \ln \left[\Gamma_{\delta, \varepsilon} \Gamma_{\delta_{1}, \delta_{2}} \Gamma_{\varepsilon_{a}, \varepsilon_{b}}\right]=d \ln \left[\frac{\left(n_{\delta_{1}}+n_{\delta_{2}}+n_{\varepsilon_{a}}+n_{\varepsilon_{b}}\right) !}{n_{\delta_{1}} ! n_{\delta_{2}} ! n_{\varepsilon_{a}} ! n_{\varepsilon_{b}} !}\right] .
$$

For large values of $n_{\delta_{1}}, n_{\delta_{2}}, n_{\varepsilon_{a}}, n_{\varepsilon_{b}}$, and using the Stirling's formula $\ln n ! \simeq n \ln n-n$, the entropy $\tilde{S}_{\Gamma}$ has the form (21). The comparison tells us that the fractal dimensions of the colliding objects and the fractal dimensions of the fragmentation processes in the final state are constituted from quanta.

The quantum conditions (20) for the fractal dimensions allow us to write the conservation law for the fractal cumulativity $C(D, \zeta)$ in units of the dimensional quantum $d$ as follows

$$
\frac{n_{\delta_{1}} x_{1}}{\left(1-x_{1}\right)}+\frac{n_{\delta_{2}} x_{2}}{\left(1-x_{2}\right)}=\frac{n_{\varepsilon_{a}} y_{a}}{\left(1-y_{a}\right)}+\frac{n_{\varepsilon_{b}} y_{b}}{\left(1-y_{b}\right)}
$$

The preservation of the number of quanta of fractal cumulativity is expected to be valid at any resolution given by arbitrary momenta $P_{1}, P_{2}$ and $p$ of the colliding and inclusive particles.

We have illustrated that the $z$-scaling can be a tool to search for and study of new symmetries, conservation laws and quantum properties of hadron structure and fragmentation processes especially at small distances. The measurements of particle spectra with high transverse momenta at the energies of the future accelerators FAIR (GSI) and NICA (JINR) will be extremely suitable for studying the regime of large fractal cumulativities and could contribute to a verification of the quantum nature of fractality in the interactions of hadrons and nuclei.

Acknowledgments. This work was supported by the MEYS of the Czech Republic under the contract LTT17018.

\section{References}

[1] I. Zborovský and M.V. Tokarev, Phys. Rev. D 75, 094008 (2007)

[2] I. Zborovský and M.V. Tokarev, Int. J. Mod. Phys. A 24, 1417 (2009) 
[3] M.V. Tokarev and I. Zborovský, J. Mod. Phys. 3, 815 (2012)

[4] M.V. Tokarev, I. Zborovský and A.A. Aparin, Phys. Part. Nucl. Lett. 12, 48 (2015)

[5] M.V. Tokarev and I. Zborovský, Int. J. Mod. Phys. A 32, 1750029 (2017)

[6] M.V. Tokarev and I. Zborovský, Phys. At. Nucl. 72, 588 (2009)

[7] M.V. Tokarev and I. Zborovský, Phys. Part. Nucl. Lett. 7, 160 (2010)

[8] M.V. Tokarev and I. Zborovský, Phys. Part. Nucl. Lett. 7, 171 (2010)

[9] M.V. Tokarev at al., Phys. Part. Nucl. Lett. 8, 533 (2011)

[10] STAR Collab. (M.V. Tokarev), Phys. At. Nucl. 74, 799 (2011)

[11] M.V. Tokarev and I. Zborovský, Phys. At. Nucl. 75, 700 (2012)

[12] M.V. Tokarev, I. Zborovský and A.A. Aparin, Phys. Part. Nucl. Lett. 12, 221 (2015)

[13] M.V. Tokarev and I. Zborovský, Theor. Math. Phys. 184, 1350 (2015)

[14] I. Zborovský, Int. J. Mod. Phys. A 33, 1850057 (2018) 